\title{
Multiple Lump Novel and Accurate Analytical and Numerical Solutions of the Three-Dimensional Potential Yu-Toda-Sasa-Fukuyama Equation
}

\author{
Mostafa M. A. Khater ${ }^{1,2, *,+} \mathbb{D}$, Dumitru Baleanu ${ }^{3,+}$ and Mohamed S. Mohamed ${ }^{4,5,+}$ (iD) \\ 1 Department of Mathematics, Faculty of Science, Jiangsu University, Zhenjiang 212013, China \\ Department of Mathematics, Obour Institutes, Cairo 11828, Egypt \\ 3 Institute of Space Sciences, 77125 Magurele-Bucharest, Romania; cankaya@edu.tr \\ 4 Department of Mathematics, Faculty of Science, Taif University, P.O. Box 11099, Taif 21944, Saudi Arabia; \\ m.saaad@tu.edu.sa \\ 5 Department of Mathematics, Faculty of Science, Al-Azher University, Nasr City, Cairo 11884, Egypt \\ * Correspondence: mostafa.khater2024@yahoo.com or 1000005364@ujs.edu.cn \\ + These authors contributed equally to this work.
}

Received: 17 November 2020; Accepted: 8 December 2020; Published: 15 December 2020

\begin{abstract}
The accuracy of novel lump solutions of the potential form of the three-dimensional potential Yu-Toda-Sasa-Fukuyama (3-Dp-YTSF) equation is investigated. These solutions are obtained by employing the extended simplest equation (ESE) and modified Kudryashov (MKud) schemes to explore its lump and breather wave solutions that characterizes the dynamics of solitons and nonlinear waves in weakly dispersive media, plasma physics, and fluid dynamics. The accuracy of the obtained analytical solutions is investigated through the perspective of numerical and semi-analytical strategies (septic B-spline (SBS) and variational iteration (VI) techniques). Additionally, matching the analytical and numerical solutions is represented along with some distinct types of sketches. The superiority of the MKud is showed as the fourth research paper in our series that has been beginning by Mostafa M. A. Khater and Carlo Cattani with the title "Accuracy of computational schemes". The functioning of employed schemes appears their effectual and ability to apply to different nonlinear evolution equations.
\end{abstract}

Keywords: three-dimensional potential Yu-Toda-Sasa-Fukuyama (3-Dp-YTSF) equation; Analytical; semi-analytical; numerical solutions

\section{Introduction}

Soliton is one of the most well-known properties that characterize the integrability of nonlinear evolution equations [1,2]. Recently, the rogue waves or monster waves, freak waves, abnormal waves have been observed as one of the soliton waves which are an exceptional type of nonlinear waves that are restricted in just one direction and have much significance in a variety of physical branches [3,4]. These waves are first figured out in the deep ocean $[5,6]$. The applications of this wave appear in the oceanography and optical fibers [7-9]. Nowadays, a new branch of the rogue wave is known as a lump wave which is defined by a restricted rogue wave in every direction in space [10-12].

Nowadays and based on computer revolution that has a great effect on deriving computational, semi-analytical and numerical schemes, many schemes have derived such as sech-tanh expansion method, auxiliary equation method, direct algebraic equation method, iteration method, exponential expansion method, B-spline schemes, Kudryashov methods, Adomian decomposition method, Khater methods, $\left(\frac{\varphi^{\prime}(\zeta)}{\varphi(\zeta)}\right)$-expansion methods and so on [13-19], the lump solutions of many nonlinear phenomena have been investigated [20-24]. 
In this context, our paper aims to investigate novel lump solutions of the 3-Dp-YTSF equation through the perspective of ESE and MKud analytical techniques [25,26]. Additionally, The another goal of this manuscript limits to check semi-analytical and numerical solutions of the considered model to explain the accuracy of the obtained analytical solutions and employed analytical schemes $[27,28]$. The 3-Dp-YTSF equation is given by [29-34]

$$
-\mathcal{Y}_{x t}+\mathcal{Y}_{x x x z}+4 \mathcal{Y}_{x} \mathcal{Y}_{x z}+2 \mathcal{Y}_{x x} \mathcal{Y}_{z}+3 \mathcal{Y}_{y y}=0
$$

where $\mathcal{Y}=\mathcal{Y}(\zeta, t), \zeta=(x, y, z)$ explains the dynamics of solitons and nonlinear waves. Employing the next wave transformation $\mathcal{Y}=\mathcal{Y}(x, y, z, t)=\mathcal{T}(\varphi)$ where $\varphi=\delta+\lambda t, \delta=x+y+z$ converts Equation (1) into the following ordinary differential equation. Integrating the obtained equation once with zero constant of integration, get

$$
(3-\lambda) \mathcal{T}^{\prime}+\mathcal{T}^{\prime \prime \prime}+3\left(\mathcal{T}^{\prime}\right)^{2}=0
$$

Handling Equation (2) through the perspective of the above-mentioned analytical schemes and the homogeneous balance principles, gives $n=1$. Thus, the general solutions of Equation (2) are evaluated by

$$
\mathcal{T}(\varphi)=\left\{\begin{array}{c}
\sum_{i=-n}^{n} a_{i} \mathcal{C}(\varphi)^{i}=a_{1} \mathcal{C}(\varphi)+\frac{a_{-1}}{\mathcal{C}(\varphi)}+a_{0} \\
\sum_{i=0}^{n} a_{i} \mathcal{L}(\varphi)^{i}=a_{1} \mathcal{L}(\varphi)+a_{0}
\end{array}\right.
$$

where $a_{0}, a_{1}, a_{-1}$ are arbitrary constants.

The structure of the other sections in this manuscript are given as follows; Section 2 investigates lump solutions of the 3-Dp-YTSF model. Additionally, checking the accuracy of the obtained solution through the above-mentioned semi-analytical and numerical schemes. Section 3 explains the obtained solutions and achieved goals of our research paper. Section 4 gives a summary of the manuscript.

\section{Analytical and Numerical Matching for the 3-Dp-YTSF Equation}

This section investigates the analytical, semi-analytical and numerical simulations for the 3-Dp-YTSF equation. The headline of this section can be summarized in the following order:

- Applying ESE and MKud schemes to Equation (2) to get lump wave solutions then getting the initial and boundary conditions to investigate semi-analytical and numerical solutions trough the SBS and VI techniques.

- Checking the accuracy of the obtained solutions along calculating the absolute value of error between exact and semi-analytical \& numerical solutions.

\subsection{ESE Analytical vs. SBS and VI Numerical Techniques along 3-Dp-YTSF Equation}

Applying the ESE scheme's framework and its auxiliary $\left(\mathcal{C}^{\prime}(\varphi)=h_{3} \mathcal{C}(\varphi)^{2}+h_{2} \mathcal{C}(\varphi)+h_{1}\right)$, where $h_{i}, i=1,2,3$ are arbitrary constants to be determined later, to Equation (2), obtains the following sets of the above-mentioned parameters:

Set I

$$
a_{-1} \rightarrow 2 h_{1}, a_{1} \rightarrow 0, \lambda \rightarrow h_{2}^{2}-4 h_{1} h_{3}+3 .
$$

Set II

$$
a_{-1} \rightarrow 0, a_{1} \rightarrow-2 h_{3}, \lambda \rightarrow h_{2}^{2}-4 h_{1} h_{3}+3
$$


Therefore, the solitary solutions of $(2+1) \mathrm{D}-3$-Dp-YTSF equation are constructed in the following formulas.

For $h_{2}=0, h_{1} h_{3}>0$, we get

$$
\begin{aligned}
& \mathcal{Y}_{\mathrm{I}, 1}(\zeta, t)=a_{0}+2 \sqrt{h_{1} h_{3}} \cot \left(\sqrt{h_{1} h_{3}}\left(\eta+\left(3-4 h_{1} h_{3}\right) t+\delta\right)\right), \\
& \mathcal{Y}_{\mathrm{I}, 2}(\zeta, t)=a_{0}+2 \sqrt{h_{1} h_{3}} \tan \left(\sqrt{h_{1} h_{3}}\left(\eta+\left(3-4 h_{1} h_{3}\right) t+\delta\right)\right), \\
& \mathcal{Y}_{\mathrm{II}, 1}(\zeta, t)=a_{0}-2 \sqrt{h_{1} h_{3}} \tan \left(\sqrt{h_{1} h_{3}}\left(\eta+\left(3-4 h_{1} h_{3}\right) t+\delta\right)\right), \\
& \mathcal{Y}_{\mathrm{II}, 2}(\zeta, t)=a_{0}-2 \sqrt{h_{1} h_{3}} \cot \left(\sqrt{h_{1} h_{3}}\left(\eta+\left(3-4 h_{1} h_{3}\right) t+\delta\right)\right) .
\end{aligned}
$$

For $h_{2}=0, h_{1} h_{3}<0$, we get

$$
\begin{aligned}
& \mathcal{Y}_{\mathrm{I}, 3}(\zeta, t)=a_{0}-2 \sqrt{-h_{1} h_{3}} \operatorname{coth}\left(\sqrt{-h_{1} h_{3}}\left(\left(3-4 h_{1} h_{3}\right) t+\delta\right) \mp \frac{\log (\eta)}{2}\right), \\
& \mathcal{Y}_{\mathrm{I}, 4}(\zeta, t)=a_{0}-2 \sqrt{-h_{1} h_{3}} \tanh \left(\sqrt{-h_{1} h_{3}}\left(\left(3-4 h_{1} h_{3}\right) t+\delta\right) \mp \frac{\log (\eta)}{2}\right), \\
& \mathcal{Y}_{\mathrm{II}, 3}(\zeta, t)=a_{0}-2 \sqrt{-h_{1} h_{3}} \tanh \left(\sqrt{-h_{1} h_{3}}\left(\left(3-4 h_{1} h_{3}\right) t+\delta\right) \mp \frac{\log (\eta)}{2}\right), \\
& \mathcal{Y}_{\mathrm{II}, 4}(\zeta, t)=a_{0}-2 \sqrt{-h_{1} h_{3}} \operatorname{coth}\left(\sqrt{-h_{1} h_{3}}\left(\left(3-4 h_{1} h_{3}\right) t+\delta\right) \mp \frac{\log (\eta)}{2}\right) .
\end{aligned}
$$

For $h_{1}=0, h_{2}>0$, we get

$$
\mathcal{Y}_{\mathrm{II}, 5}(\zeta, t)=a_{0}+\frac{2 h_{2}}{h_{3} e^{h_{2}\left(\eta+\left(h_{2}^{2}+3\right) t+\delta\right)}-1}+2 h_{2} .
$$

For $h_{1}=0, h_{2}<0$, we get

$$
\mathcal{Y}_{\mathrm{II}, 6}(\zeta, t)=a_{0}-\frac{2 h_{3}}{h_{3} e^{h_{2}\left(\eta+\left(h_{2}^{2}+3\right) t+\delta\right)}+1}+2 h_{3} .
$$

For $4 h_{1} h_{3}>h_{2}^{2}$, we get

$$
\begin{gathered}
\mathcal{Y}_{\mathrm{I}, 5}(\zeta, t)=a_{0}-\frac{4 h_{1} h_{3}}{h_{2}-\sqrt{4 h_{1} h_{3}-h_{2}^{2}} \tan \left(\frac{1}{2} \sqrt{4 h_{1} h_{3}-h_{2}^{2}}\left(\eta+\left(h_{2}^{2}-4 h_{1} h_{3}+3\right) t+\delta\right)\right)}, \\
\mathcal{Y}_{\mathrm{I}, 6}(\zeta, t)=a_{0}-\frac{4 h_{1} h_{3}}{h_{2}-\sqrt{4 h_{1} h_{3}-h_{2}^{2}} \cot \left(\frac{1}{2} \sqrt{4 h_{1} h_{3}-h_{2}^{2}}\left(\eta+\left(h_{2}^{2}-4 h_{1} h_{3}+3\right) t+\delta\right)\right)}, \\
\mathcal{Y}_{\mathrm{II}, 7}(\zeta, t)=a_{0}-\sqrt{4 h_{1} h_{3}-h_{2}^{2}} \tan \left(\frac{1}{2} \sqrt{4 h_{1} h_{3}-h_{2}^{2}}\left(\eta+\left(h_{2}^{2}-4 h_{1} h_{3}+3\right) t+\delta\right)\right)+h_{2} \\
\mathcal{Y}_{\mathrm{II}, 8}(\zeta, t)=a_{0}+\sqrt{4 h_{1} h_{3}-h_{2}^{2}}\left(-\cot \left(\frac{1}{2} \sqrt{4 h_{1} h_{3}-h_{2}^{2}}\left(\eta+\left(h_{2}^{2}-4 h_{1} h_{3}+3\right) t+\delta\right)\right)\right)+h_{2} .
\end{gathered}
$$

\section{Matching between Analytical and Numerical}

Here, we employ two recent numerical and semi-analytical schemes to verify the accuracy of our analytical obtained solutions: 
- Applying the SBS numerical technique with the following initial condition $\mathcal{Y}(x, 0,0,0)=$ $-2 \tanh (x)$ gets the following shown analytical and numerical solutions' values with respect to different values of $x$ in Table 1

Table 1. Analytical and numerical matching through ESE and SBS schemes.

\begin{tabular}{cccc}
\hline Value of $x$ & Analytical & Numerical & Absolute Error \\
\hline 0 & 0 & $2.59953 \times 10^{-20}$ & $2.59953 \times 10^{-20}$ \\
0.00001 & -0.00002 & $-4.94237 \times 10^{-14}$ & 0.00002 \\
0.00002 & -0.00004 & $-6.43149 \times 10^{-14}$ & 0.00004 \\
0.00003 & -0.00006 & $-1.08723 \times 10^{-13}$ & 0.00006 \\
0.00004 & -0.00008 & $-1.13736 \times 10^{-13}$ & 0.00008 \\
0.00005 & -0.0001 & $-1.48758 \times 10^{-13}$ & 0.0001 \\
0.00006 & -0.00012 & $-1.44334 \times 10^{-13}$ & 0.00012 \\
0.00007 & -0.00014 & $-1.69924 \times 10^{-13}$ & 0.00014 \\
0.00008 & -0.00016 & $-1.56069 \times 10^{-13}$ & 0.00016 \\
0.00009 & -0.00018 & $-1.72219 \times 10^{-13}$ & 0.00018 \\
0.0001 & -0.0002 & $-1.49012 \times 10^{-13}$ & -0.0002 \\
\hline
\end{tabular}

- Applying the VI method to Equation (1), gets the following semi-analytical solutions:

$$
\mathcal{Y}_{n+1}=\mathcal{Y}_{n}-\int_{0}^{t}\left(-\left(\mathcal{Y}_{n}\right)_{x s}+\left(\mathcal{Y}_{n}\right)_{x x x z}+4\left(\mathcal{Y}_{n}\right)_{x}\left(\mathcal{Y}_{n}\right)_{x z}+2\left(\mathcal{Y}_{n}\right)_{x x}\left(\mathcal{Y}_{n}\right)_{z}+3\left(\mathcal{Y}_{n}\right)_{y y}\right) d s
$$

Using Equation (18)

$$
\begin{gathered}
\mathcal{Y}_{0}(\zeta, t)=-2 \tanh (\delta) \\
\mathcal{Y}_{1}(\zeta, t)=2 \tanh (\delta)\left(48 t \operatorname{sech}^{4}(\delta)-14 t \operatorname{sech}^{2}(\delta)-1\right), \\
\mathcal{Y}_{2}(\zeta, t)=2 \tanh (\delta)\left(4 t \operatorname { s e c h } ^ { 2 } ( \delta ) \left(\operatorname{sech}^{2}(\delta)(1404 t(9-28 t)\right.\right. \\
\times \operatorname{sech}^{2}(\delta)+48 t \operatorname{sech}^{4}(\delta)(450 t(3-13 \cosh (2(\delta))) \\
\left.\left.\left.\left.\times \operatorname{sech}^{4}(\delta)+5683 t-270\right)+t(1568 t-2475)+24\right)+49 t-7\right)-1\right) .
\end{gathered}
$$

Using same steps we can get $\mathcal{Y}_{i}, i=3,4,5, \cdots$.

\subsection{Kud Analytical vs. SBS and VI Numerical Techniques along 3-Dp-YTSF Equation}

Applying the MKud scheme's framework and its auxiliary $\left(\mathcal{L}^{\prime}(\varphi)=\ln (k)\left(\mathcal{L}(\varphi)^{2}-\mathcal{L}(\varphi)\right)\right)$, where $k$ is arbitrary constant to be evaluated later, to Equation (2), obtains the following sets of the above-mentioned parameters:

\section{Set I}

$$
a_{0}=-1, a_{1}=2, r=2, \mu=-\frac{1}{\log ^{2}(k)}
$$

\section{Set II}

$$
a_{0}=0, a_{1}=1, r=\frac{4 \log (k)-3}{2 \log (k)}, \mu=-\frac{1}{4 \log ^{2}(k)} .
$$




\section{Set III}

$$
a_{0}=1, a_{1}=-1, r=\frac{4 \log (k)+3}{2 \log (k)}, \mu=-\frac{1}{4 \log ^{2}(k)} .
$$

Therefore, the solitary solutions of 3-Dp-YTSF equation are constructed in the following formulas:

$$
\mathcal{Y}(\zeta, t)=a_{0}-\frac{2 \log (k)}{1 \pm k^{t\left(\log ^{2}(k)+3\right)+\delta}} .
$$

Matching between Analytical, Semi-Analytical and Numerical Solutions

Here, we employ two recent numerical and semi-analytical schemes to verify the accuracy of our analytical obtained solutions:

- $\quad$ Applying the SBS numerical technique with the following initial condition $\mathcal{Y}(x, 0,0,0)=\tanh \left(\frac{x}{2}\right)$ gets the following shown analytical and numerical solutions' values with respect to different values of $x$ in Table 2

Table 2. Analytical and numerical matching through MKud and SBS schemes.

\begin{tabular}{cccc}
\hline Value of $x$ & Analytical & Numerical & Error \\
\hline 0 & 0 & $2.98907 \times 10^{-21}$ & $2.98907 \times 10^{-21}$ \\
0.00001 & $5 \times 10^{-6}$ & $1.02496 \times 10^{-13}$ & $5 \times 10^{-6}$ \\
0.00002 & 0.00001 & $1.31139 \times 10^{-13}$ & 0.00001 \\
0.00003 & 0.000015 & $2.19425 \times 10^{-13}$ & 0.000015 \\
0.00004 & 0.00002 & $2.28909 \times 10^{-13}$ & 0.00002 \\
0.00005 & 0.000025 & $2.98536 \times 10^{-13}$ & 0.000025 \\
0.00006 & 0.00003 & $2.89309 \times 10^{-13}$ & 0.00003 \\
0.00007 & 0.000035 & $3.40231 \times 10^{-13}$ & 0.000035 \\
0.00008 & 0.00004 & $3.12301 \times 10^{-13}$ & 0.00004 \\
0.00009 & 0.000045 & $3.44501 \times 10^{-13}$ & 0.000045 \\
0.0001 & 0.00005 & $2.98023 \times 10^{-13}$ & 0.00005 \\
\hline
\end{tabular}

- $\quad$ Applying VI method along Equation (18) to Equation (1), obtains

$$
\begin{gathered}
\mathcal{Y}_{0}(\zeta, t)=\tanh \left(\frac{1}{2}(\delta)\right) \\
\mathcal{Y}_{1}(\zeta, t)=\frac{1}{2} \tanh \left(\frac{1}{2}(\delta)\right) \operatorname{sech}^{2}\left(\frac{1}{2}(\delta)\right)(4 t+\cosh (\delta)+1) \\
\mathcal{Y}_{2}(\zeta, t)=\frac{1}{128} \tanh \left(\frac{1}{2}(\delta)\right) \operatorname{sech}^{8}\left(\frac{1}{2}(\delta)\right)\left(4\left(8 t\left(4 t^{2}+6 t+3\right)+7\right)\right. \\
\times \cosh (2(\delta))+32 t\left(84 t^{2}+4 t+5\right)+8(2 t(7 t(3-16 t)+15)+7) \\
\times \cosh (\delta)+8(1-2(t-1) t) \cosh (3(\delta))+\cosh (4(\delta))+35)
\end{gathered}
$$

Using same steps we can get $\mathcal{Y}_{i}, i=3,4,5, \cdots$.

\section{Results' Interpretation}

This section studies the originality and novelty of our research paper. It also shows the accuracy of the obtained analytical solutions. The ESE and MKud computational schemes were applied to the 3-Dp-YTSF equation for constructed solitary wave solutions. Many distinct wave solutions have 
been obtained, and some of them were demonstrated through some sketches in 2D, 3D, and contour plots to explain the dynamic behaviour of the soliton waves in weakly dispersive media. Comparing our solutions with those that were obtained in previously published papers shows our solutions are completely different from that have been evaluated in [29-33], but some of our solutions are matching the obtained solutions in [34] when $\left(a_{0}=\left(A_{0}+1\right), h_{1} h_{3}=\frac{-\left(3 l^{2}+4 c\right)}{4 m}, c=\lambda, \eta=1\right)$ Equation (33) [34] are equal to Equation (9), however the authors in that paper have studied the same model with modified simplest equation method.

The SBS and VI schemes have been applied to the considered model based on the obtained computational solutions. The absolute error between the analytical and numerical solutions was calculated to show the accuracy of the solutions and used methods (Tables 1 and 2; Figures 1 and 2) while Figure 3 shows lump solitary wave solutions. While Figure 4 represents the matching between analytical and numerical solutions of Equation (1) based on Table 2. Additionally, Figure 5 demonstrates the semi-analytical solutions of Equation (1) in two and three-dimensional plots. This calculating shows the arrogance of the MKud method over the ESE method where its absolute values of error are much smaller than that have been obtained by the ESE method (Figure 6).
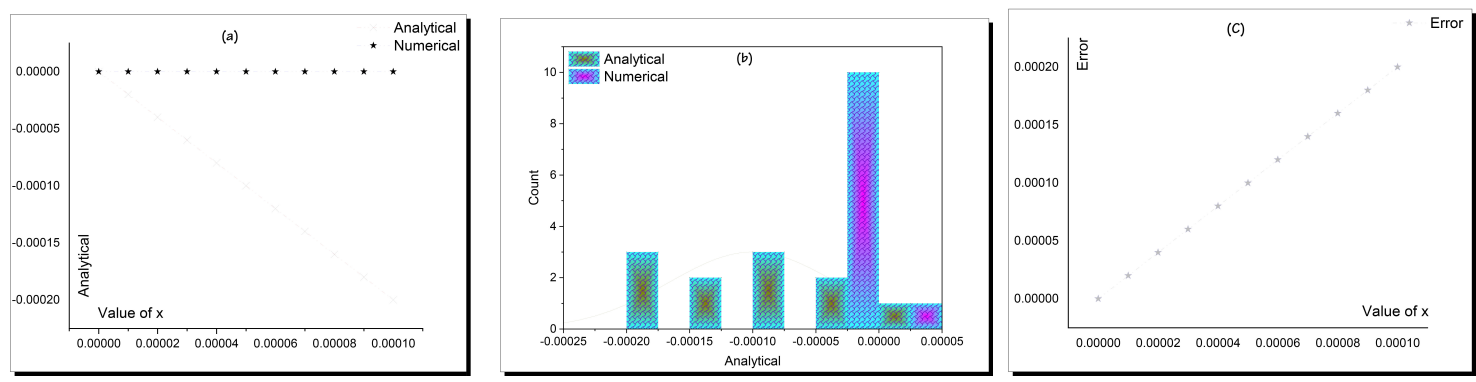

Figure 1. Analytical, numerical represented in two dimensional (a), column plot (b), while absolute error for Equation (1) is represented in two dimensional (c) with respect to Table 1.
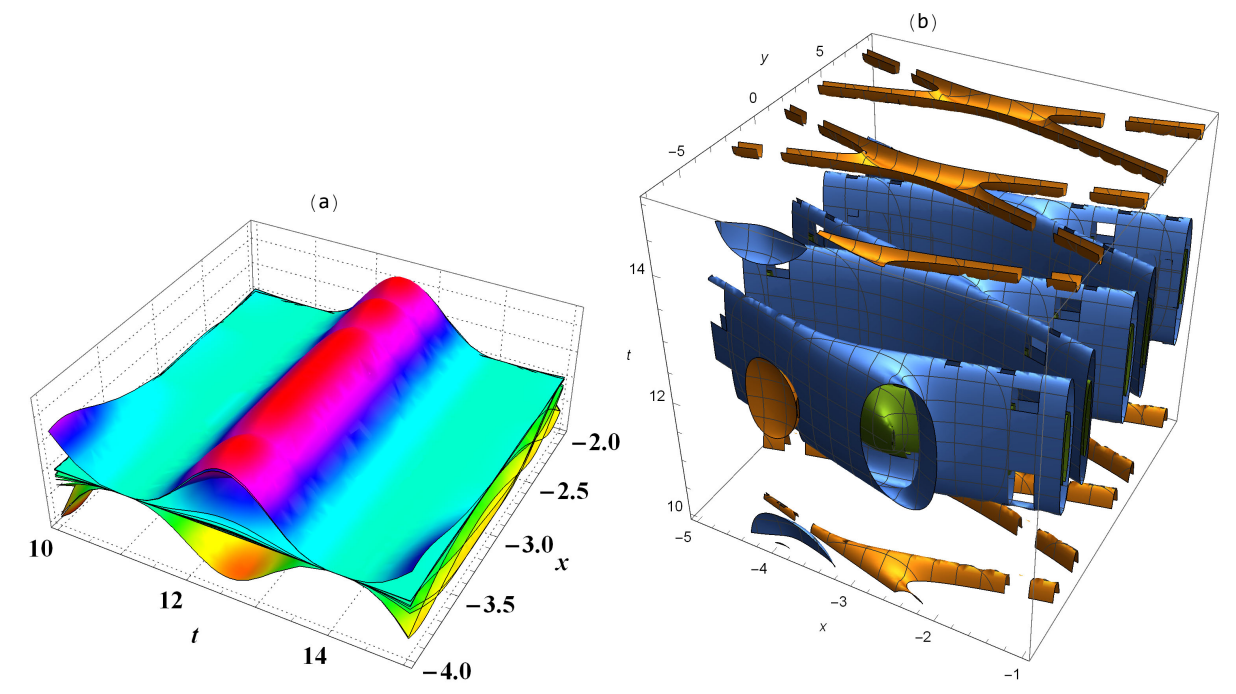

Figure 2. Semi-analytical solutions of Equation (2) in three-dimensional (a) and contour plots (b). 

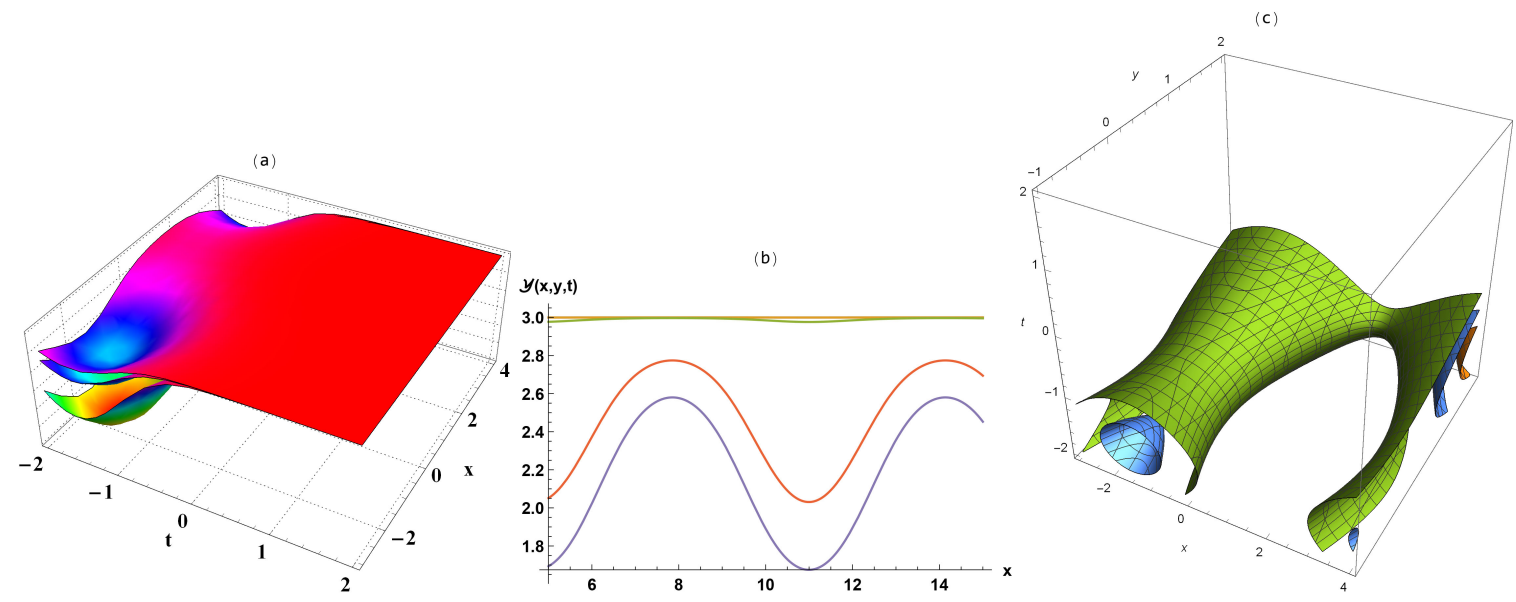

Figure 3. Solitary wave solutions Equation (22) in three (a), two (b), and contour plots (c).
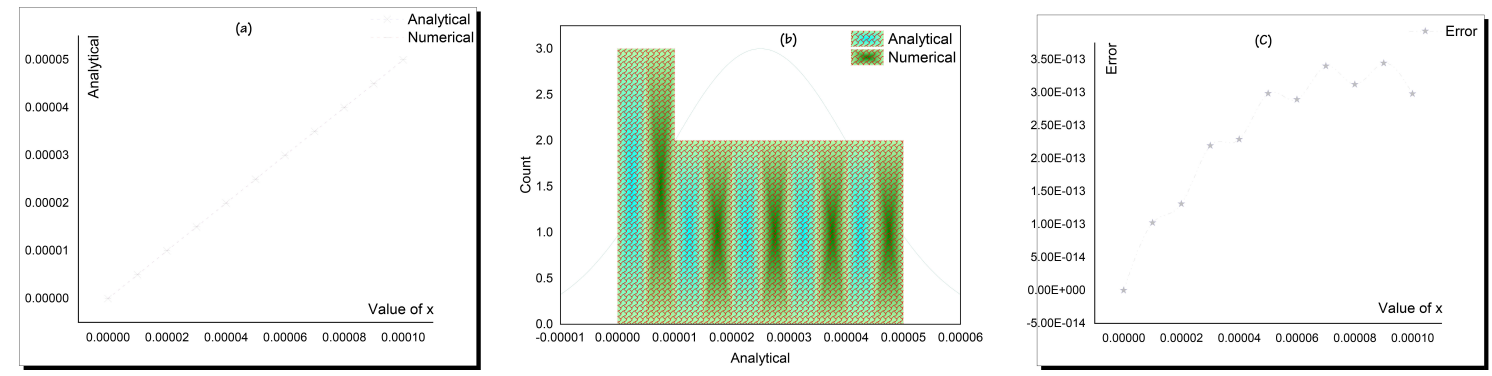

Figure 4. Analytical, numerical represented in two dimensional (a), column plot (b), while absolute error for Equation (1) is represented in two dimensional (c) with respect to Table 2.

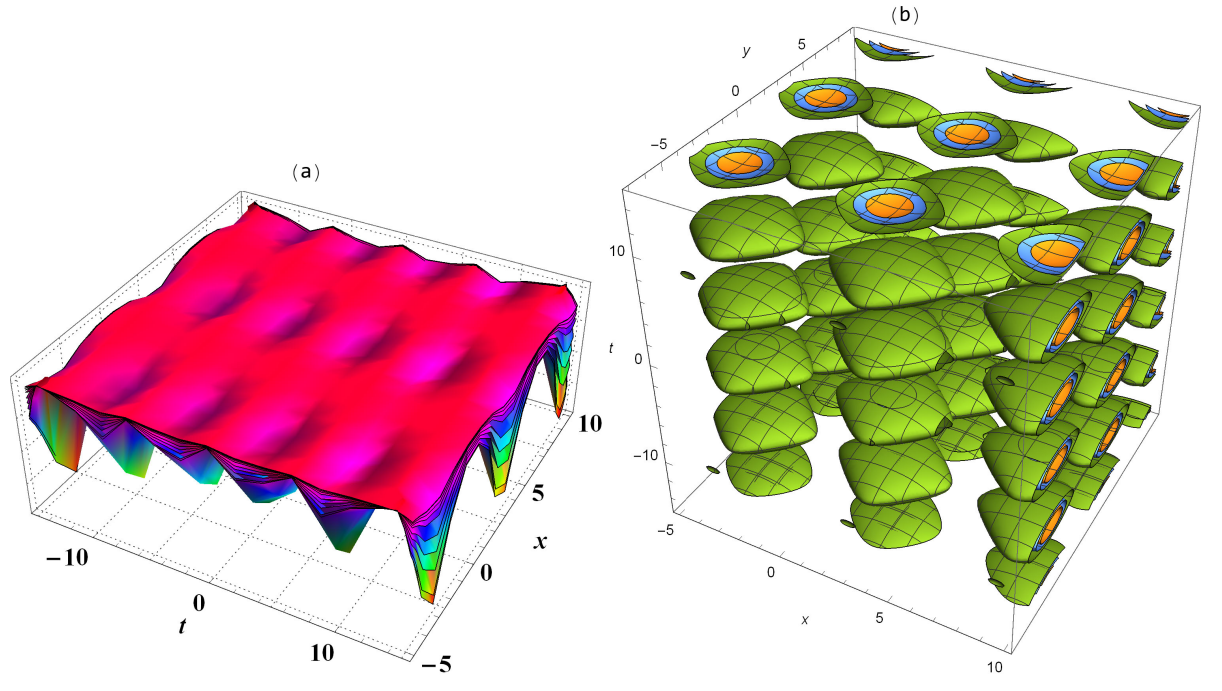

Figure 5. Semi-analytical solutions of Equation (1) in three-dimensional (a) and contour plots (b). 

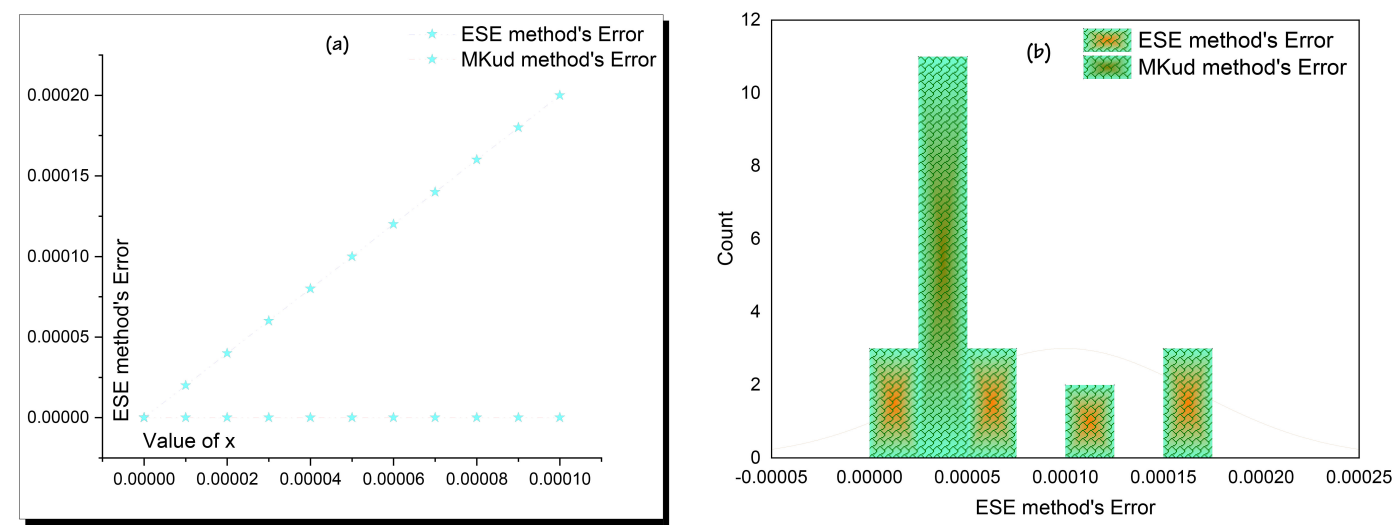

Figure 6. Absolute error along ESE, MKud analytical schemes and TQBS numerical scheme in two dimensional (a) and column plots (b).

\section{Conclusions}

This research paper successfully handled the nonlinear 3-Dp-YTSF equation arising in fluid dynamics, plasma physics and weakly dispersive media. The ESE, MKud, SBS, and VI schemes were employed to find accurate novel solitary wave solutions of the considered model. Lump wave solutions have been represented in three various types of sketches. The accuracy of the MKud method has been verified over the ESE method where it is our fourth paper in series with title accuracy of the computational schemes. This series aims to determine the accuracy of all computational schemes.

Author Contributions: The authors conceived of the study, M.M.A.K. participated in its design and coordination, D.B. participated in drafted the manuscript, M.S.M. participated in the sequence alignment, M.M.A.K., D.B. and M.S.M. participated in reading and approved the final manuscript. All authors have read and agreed to the published version of the manuscript.

Funding: This paper was funded by “Taif University Researchers Supporting Project number (TURSP-2020/160), Taif University, Taif, Saudi Arabia".

Acknowledgments: The authors are thankful of the Taif University. Taif University researchers supporting project number (TURSP-2020/160), Taif University, Taif, Saudi Arabia.

Conflicts of Interest: The authors declare that we have no competing interest.

Availability of Data and Materials: The data that support the findings of this study are available from the corresponding author upon reasonable request.

\section{References}

1. Shen, B.; Chang, L.; Liu, J.; Wang, H.; Yang, Q.F.; Xiang, C.; Wang, R.N.; He, J.; Liu, T.; Xie, W.; et al. Integrated turnkey soliton microcombs. Nature 2020, 582, 365-369. [CrossRef] [PubMed]

2. Ni, G.; Wang, H.; Jiang, B.Y.; Chen, L.; Du, Y.; Sun, Z.; Goldflam, M.; Frenzel, A.; Xie, X.; Fogler, M.; et al. Soliton superlattices in twisted hexagonal boron nitride. Nat. Commun. 2019, 10, 1-6. [CrossRef] [PubMed]

3. Dudley, J.M.; Genty, G.; Mussot, A.; Chabchoub, A.; Dias, F. Rogue waves and analogies in optics and oceanography. Nat. Rev. Phys. 2019, 1, 675-689. [CrossRef]

4. Su, J.J.; Gao, Y.T.; Deng, G.F.; Jia, T.T. Solitary waves, breathers, and rogue waves modulated by long waves for a model of a baroclinic shear flow. Phys. Rev. E 2019, 100, 042210. [CrossRef]

5. Dematteis, G.; Grafke, T.; Onorato, M.; Vanden-Eijnden, E. Experimental evidence of hydrodynamic instantons: The universal route to rogue waves. Phys. Rev. X 2019, 9, 041057. [CrossRef]

6. Kannan, R.; Wang, Z. A high order spectral volume solution to the Burgers' equation using the Hopf-Cole transformation. Int. J. Numer. Methods Fluids 2012, 69, 781-801. [CrossRef]

7. Lan, Z.Z.; Su, J.J. Solitary and rogue waves with controllable backgrounds for the non-autonomous generalized AB system. Nonlinear Dyn. 2019, 96, 2535-2546. [CrossRef]

8. Marcucci, G.; Pierangeli, D.; Conti, C. Theory of neuromorphic computing by waves: Machine learning by rogue waves, dispersive shocks, and solitons. Phys. Rev. Lett. 2020, 125, 093901. [CrossRef] 
9. Kannan, R. A high order spectral volume formulation for solving equations containing higher spatial derivative terms: Formulation and analysis for third derivative spatial terms using the LDG discretization procedure. Commun. Comput. Phys. 2011, 10, 1257-1279. [CrossRef]

10. Ilhan, O.A.; Manafian, J.; Shahriari, M. Lump wave solutions and the interaction phenomenon for a variable-coefficient Kadomtsev-Petviashvili equation. Comput. Math. Appl. 2019, 78, 2429-2448. [CrossRef]

11. Kannan, R. A high order spectral volume formulation for solving equations containing higher spatial derivative terms II: Improving the third derivative spatial discretization using the LDG2 method. Commun. Comput. Phys. 2012, 12, 767-788. [CrossRef]

12. Kannan, R.; Wang, Z.J. LDG2: A variant of the LDG flux formulation for the spectral volume method. J. Sci. Comput. 2011, 46, 314-328. [CrossRef]

13. Khater, M.M.; Baleanu, D. On abundant new solutions of two fractional complex models. Adv. Differ. Equ. 2020, 2020, 1-14. [CrossRef]

14. Abdel-Aty, A.H.; Khater, M.M.; Dutta, H.; Bouslimi, J.; Omri, M. Computational solutions of the HIV-1 infection of CD4+ T-cells fractional mathematical model that causes acquired immunodeficiency syndrome (AIDS) with the effect of antiviral drug therapy. Chaos Solitons Fractals 2020, 139, 110092. [CrossRef]

15. Khater, M.M.; Attia, R.A.; Park, C.; Lu, D. On the numerical investigation of the interaction in plasma between (high \& low) frequency of (Langmuir \& ion-acoustic) waves. Results Phys. 2020, 18, 103317.

16. Qin, H.; Khater, M.; Attia, R.A. Inelastic Interaction and Blowup New Solutions of Nonlinear and Dispersive Long Gravity Waves. J. Funct. Spaces 2020, 2020, 5362989. [CrossRef]

17. Khater, M.M.; Ghanbari, B.; Nisar, K.S.; Kumar, D. Novel exact solutions of the fractional Bogoyavlensky-Konopelchenko equation involving the Atangana-Baleanu-Riemann derivative. Alex. Eng. J. 2020, 59, 2957-2967. [CrossRef]

18. Yue, C.; Lu, D.; Khater, M.M.; Abdel-Aty, A.H.; Alharbi, W.; Attia, R.A. On explicit wave solutions of the fractional nonlinear DSW system via the modified Khater method. Fractals 2020. [CrossRef]

19. Abdel-Aty, A.H.; Khater, M.; Attia, R.A.; Eleuch, H. Exact Traveling and Nano-Solitons Wave Solitons of the Ionic Waves Propagating along Microtubules in Living Cells. Mathematics 2020, 8, 697. [CrossRef]

20. Qin, H.; Khater, M.; Attia, R.A. Copious Closed Forms of Solutions for the Fractional Nonlinear Longitudinal Strain Wave Equation in Microstructured Solids. Math. Probl. Eng. 2020, 2020, 3498796. [CrossRef]

21. Li, J.; Attia, R.A.; Khater, M.M.; Lu, D. The new structure of analytical and semi-analytical solutions of the longitudinal plasma wave equation in a magneto-electro-elastic circular rod. Mod. Phys. Lett. B 2020, 34, 2050123. [CrossRef]

22. Khater, M.M.; Attia, R.A.; Alodhaibi, S.S.; Lu, D. Novel soliton waves of two fluid nonlinear evolutions models in the view of computational scheme. Int. J. Mod. Phys. B 2020, 34, 2050096. [CrossRef]

23. Günerhan, H.; Khodadad, F.S.; Rezazadeh, H.; Khater, M.M. Exact optical solutions of the $(2+1)$ dimensions Kundu-Mukherjee-Naskar model via the new extended direct algebraic method. Mod. Phys. Lett. B 2020, 34, 2050225. [CrossRef]

24. Yue, C.; Khater, M.M.; Inc, M.; Attia, R.A.; Lu, D. Abundant analytical solutions of the fractional nonlinear (2+ 1)-dimensional BLMP equation arising in incompressible fluid. Int. J. Mod. Phys. B 2020, 34, 2050084. [CrossRef]

25. Yue, C.; Elmoasry, A.; Khater, M.; Osman, M.; Attia, R.; Lu, D.; Elazab, N.S. On complex wave structures related to the nonlinear long-short wave interaction system: Analytical and numerical techniques. AIP Adv. 2020, 10, 045212. [CrossRef]

26. Yue, C.; Khater, M.M.; Attia, R.A.; Lu, D. Computational simulations of the couple Boiti-Leon-Pempinelli (BLP) system and the (3+1)-dimensional Kadomtsev-Petviashvili (KP) equation. AIP Adv. 2020, 10, 045216. [CrossRef]

27. Khater, M.M.; Attia, R.A.; Lu, D. Computational and numerical simulations for the nonlinear fractional Kolmogorov-Petrovskii-Piskunov (FKPP) equation. Phys. Scr. 2020, 95, 055213. [CrossRef]

28. Khater, M.M.; Alzaidi, J.; Attia, R.A.; Lu, D. Analytical and numerical solutions for the current and voltage model on an electrical transmission line with time and distance. Phys. Scr. 2020, 95, 055206. [CrossRef]

29. Dong, M.J.; Tian, S.F.; Wang, X.B.; Zhang, T.T. Lump-type solutions and interaction solutions in the (3+ 1)-dimensional potential Yu-Toda-Sasa-Fukuyama equation. Anal. Math. Phys. 2019, 9, 1511-1523. [CrossRef] 
30. Hu, Y.; Chen, H.; Dai, Z. New kink multi-soliton solutions for the (3+ 1)-dimensional potential-Yu-Toda-SasaFukuyama equation. Appl. Math. Comput. 2014, 234, 548-556. [CrossRef]

31. Zhang, S.; Zhang, H.Q. A transformed rational function method for (3+ 1)-dimensional potential Yu-TodaSasa-Fukuyama equation. Pramana 2011, 76, 561-571. [CrossRef]

32. Liu, W. Rogue waves of the (3+ 1)-dimensional potential Yu-Toda-Sasa-Fukuyama equation. Rom. Rep. Phys. 2017, 69, 16.

33. Roshid, H.O. Lump solutions to a (3+ 1)-dimensional potential-Yu-Toda-Sasa-Fukuyama (YTSF) like equation. Int. J. Appl. Comput. Math. 2017, 3, 1455-1461. [CrossRef]

34. Zayed, E.; Arnous, A. Exact solutions of the nonlinear ZK-MEW and the potential YTSF equations using the modified simple equation method. AIP Conf. Proc. 2012, 1479, 2044-2048.

Publisher's Note: MDPI stays neutral with regard to jurisdictional claims in published maps and institutional affiliations.

(C) 2020 by the authors. Licensee MDPI, Basel, Switzerland. This article is an open access article distributed under the terms and conditions of the Creative Commons Attribution (CC BY) license (http:// creativecommons.org/licenses/by/4.0/). 